\title{
Universiteit
}

Leiden

The Netherlands

\section{Raman and AFM-IR chemical imaging of stratum corneum model membranes}

Ramos, A.P.; Gooris, G.S.; Bouwstra, J.A.; Molinari, M.; Lafleur, M.

\section{Citation}

Ramos, A. P., Gooris, G. S., Bouwstra, J. A., Molinari, M., \& Lafleur, M. (2020). Raman and AFM-IR chemical imaging of stratum corneum model membranes. Canadian Journal of Chemistry, 98, 495-501. doi:10.1139/cjc-2019-0471

Version: $\quad$ Publisher's Version

License: $\quad$ Leiden University Non-exclusive license

Downloaded from: https://hdl.handle.net/1887/3201066

Note: To cite this publication please use the final published version (if applicable). 


\title{
Raman and AFM-IR chemical imaging of stratum corneum model membranes
}

\author{
Adrian Paz Ramos, Gert Gooris, Joke Bouwstra, Michael Molinari, and Michel Lafleur
}

\begin{abstract}
Stratum corneum (SC), the outermost layer of the epidermis, is the primary barrier to percutaneous absorption. The diffusion of substances through the skin occurs through the SC lipid fraction, which is essentially constituted of an equimolar mixture of ceramides, free fatty acids, and cholesterol. The lipid constituents of SC are mainly forming continuous multilamellar membranes in the solid/crystalline state. However, recent findings suggest the presence of a highly disordered (liquid) phase formed by the unsaturated C18 chain of ceramide EOS, surrounded by a highly ordered lipid environment. The aim of the present work was to study the lipid spatial distribution of model SC membranes composed of ceramide EOS, ceramide NS, a mixture of free fatty acids, and cholesterol, using Raman microspectroscopy and AFM-IR spectroscopy techniques. The enhanced spatial resolution at the tens of nanometers scale of the AFM-IR technique revealed that the lipid matrix is overall homogeneous, with the presence of small, slightly enriched, and depleted regions in a lipid component. No liquid domains of ceramide EOS were observed at this scale, a result that is consistent with the model proposing that the oleate nanodrops are concentrated in the central layer of the three-layer organization of the SC membranes forming the long periodicity phase. In addition, both Raman microspectroscopy and AFM-IR techniques confirmed the fluid nature of the unsaturated chain of ceramide EOS while the rest of the lipid matrix was found highly ordered.
\end{abstract}

Key words: lipids, stratum corneum, Raman spectroscopy, atomic force microscopy, IR spectroscopy.

Résumé : Le stratum corneum $(\mathrm{SC})$, la couche superficielle de l'épiderme, constitue la principale barrière à l'absorption percutanée. Les substances diffusent à travers la peau en passant par la fraction lipidique du SC, qui consiste essentiellement en un mélange équimolaire de céramides, d'acides gras libres et de cholestérol. Les constituants lipidiques du SC forment principalement des membranes multilamellaires continues à l'état solide/cristallin. Cependant, de récentes découvertes semblent indiquer la présence d'une phase hautement désordonnée (liquide) formée par la chaîne C18 insaturée du céramide EOS et entourée d'une phase lipidique hautement ordonnée. Le but des présents travaux était d'étudier la distribution spatiale des lipides dans des membranes modèles de SC composées de céramide EOS, de céramide NS, d'un mélange d'acides gras libres et de cholestérol, au moyen de la microspectroscopie Raman et de la spectroscopie infrarouge couplée à la microscopie à force atomique (AFM-IR). Grâce à une résolution spatiale atteignant les dizaines de nanomètres, la technique AFM-IR a révélé que la matrice lipidique était globalement homogène, mais présentait de petites zones légèrement enrichies ou appauvries en l'un des composants lipidiques. Aucun domaine liquide de céramide EOS n'a été observé à cette échelle, un résultat qui concorde avec le modèle fondé sur l'hypothèse selon laquelle les nanogouttelettes d'oléate sont concentrées dans la couche centrale des membranes à trois couches du SC qui forment la phase de longue périodicité. De plus, les techniques de microspectroscopie Raman et AFM-IR ont toutes deux permis de démontrer la nature fluide de la chaîne insaturée de céramide EOS alors que le reste de la matrice lipidique était hautement ordonnée. [Traduit par la Rédaction]

Mots-clés : lipids, stratum corneum, spectroscopie Raman, microscopie à force atomique, spectroscopie IR.

\section{Introduction}

Skin forms an impermeable and flexible interface protecting the body from the external environment. The stratum corneum (SC), the outermost layer of the epidermis, is the main reason for skin impermeability, acting as a barrier against chemical and microbiological agents, and preventing water loss from the body. SC is formed of fully keratinized dead cells (corneocytes) embedded into a complex lipid matrix. ${ }^{1}$ This lipid matrix, which is essentially constituted of an equimolar mixture of ceramides (Cer), free fatty acids (FFA), and cholesterol (Chol), forms the only continuous pathway to substances through the SC. Therefore, it plays an important role in the skin barrier function.
Human SC lipids are ordered into two coexisting lamellar phases known as the long periodicity phase (LPP), with a repeat distance of approximately $13 \mathrm{~nm}$, and the short periodicity phase (SPP), with a spacing of $6 \mathrm{~nm} .^{2,3}$ These lamellar structures are formed by laterally organized domains, an arrangement that is considered a key factor for the diffusion of substances through the SC. The SC lipids are mainly in a crystalline state, where the molecules are very closely packed, leading to a very limited permeability.

The SC lipid fraction includes more than 1000 different lipids. ${ }^{4-6}$ Among them, the $\omega$-acylceramide Cer EOS subclass is constituted by a sphingosine base bearing a very long $\omega$-hydroxy fatty acid

Received 17 December 2019. Accepted 2 March 2020.

A. Paz Ramos and M. Lafleur. Department of Chemistry, Université de Montréal, Montréal, QC H3C 3J7, Canada.

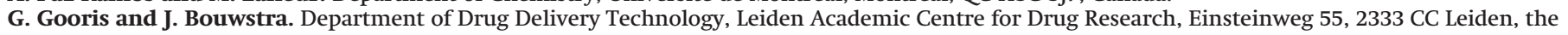
Netherlands.

M. Molinari. Institut de chimie et biologie des membranes et des nano-objets, CNRS UMR 5248, Université de Bordeaux, IPB, 33600 Pessac, France.

Corresponding author: Michel Lafleur (email: michel.lafleur@umontreal.ca).

This paper is part of a special issue celebrating the 100th anniversary of the Department of Chemistry at the Université de Montréal.

Copyright remains with the author(s) or their institution(s). Permission for reuse (free in most cases) can be obtained from RightsLink. 
Fig. 1. The molecular structure of the lipids investigated in the present study. The deuterium-labeled portions are shown in red. [Colour online.]
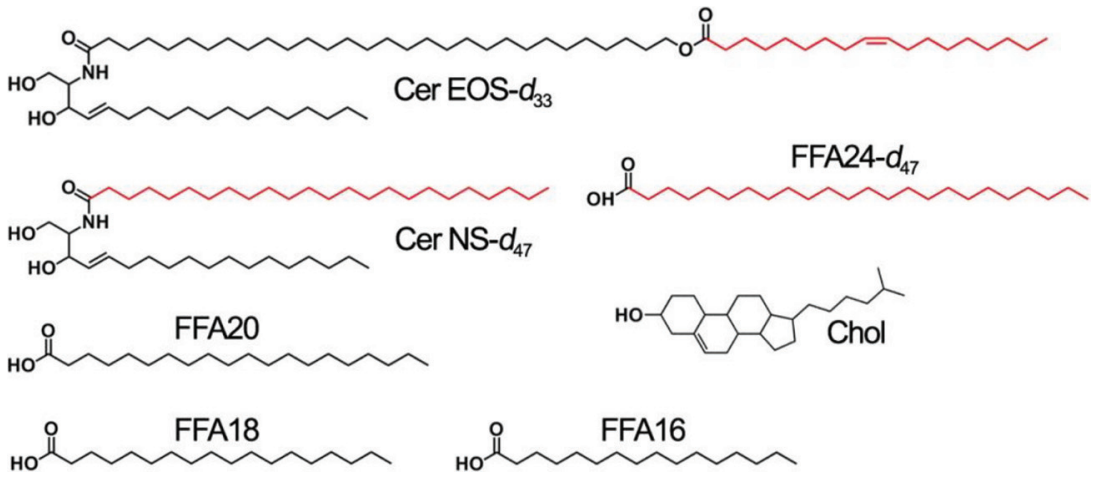

with a chain length distribution between 26 and 36 carbon atoms to which an unsaturated C18 fatty acid chain is esterified. It has been shown that Cer EOS together with Cer NS (a ceramide bearing a nonhydroxylated chain and a sphingosine base), FFAs, and cholesterol, reproduced LPP structures analogous to those observed in native SC. ${ }^{7,8}$ The formation of the LPP is crucial for ensuring the impermeability of the skin; thus, Cer EOS is considered a key component for the permeability of native and model SC membranes.

Recently, ${ }^{9}$ solid-state NMR and infrared spectroscopy measurements showed that the oleate chain of Cer EOS was highly disordered in a model mixture formed with Cer NS, Cer EOS, FFAs, and cholesterol. It was proposed that the unsaturated chain of the Cer EOS remains in a highly disordered state because of steric restrictions. At this point, it is not established how these liquid chains are integrated into the solid SC lipid matrix. A model, derived from neutron diffraction measurements, proposed that the unsaturated chains of Cer EOS are localized in the central layer, partly within the inner headgroup region, of a three-layer structure that forms the LPP. ${ }^{10}$ This lipid structure would include a solid/crystalline lipid matrix in which are embedded small hydrocarbon nanodrops formed by the oleate chains; whether these chains are isolated or grouped together remains unanswered. ${ }^{9}$ This model was recently supported by molecular dynamics simulations. ${ }^{11}$ It was observed that Cer EOS linoleate chain in the LPP undergoes transitions from straight to hooked conformations on the order of nanoseconds, leading to high disorder. At this point, there has been no direct observation of these liquid domains in the SC lipid matrix.

Raman microspectroscopy is one of the most commonly used techniques for the visualization of the lipid spatial distribution in SC model membranes. The chemical imaging is based on the identification of functional groups that allows the localization of the different lipid molecules. The Raman images can be created to describe the local lipid composition of voxels and, therefore, the heterogeneity or homogeneity of the SC lipid model mixtures. Generally, the introduction of a deuterated species facilitates this analysis because of the isotopic effect; the most common approach is the introduction of a lipid with deuterated chains, as the $\mathrm{CH}$ stretching $(\nu \mathrm{CH})$ region $\left(2780-3100 \mathrm{~cm}^{-1}\right)$ and the $\mathrm{CD}$ stretching $(\nu \mathrm{CD})$ region $\left(2000-2250 \mathrm{~cm}^{-1}\right)$ are easily resolved in the Raman spectra. ${ }^{12}$

In chemical imaging, the spatial resolution is indeed a critical factor, as it defines the limit of domain size that could be detected. The lateral resolution (d) in Raman microspectroscopy, essentially determined by the Rayleigh equation, ${ }^{13}$ depends on the laser wavelength and on the numerical aperture (NA) of the collection objective. The resulting lateral resolution is usually between 400 and $900 \mathrm{~nm} .{ }^{13}$ The depth resolution, depending on the laser wavelength, the objectives, and the optical properties of the sample varies from around $1 \mu \mathrm{m}$ to several micrometers. ${ }^{14}$
AFM-IR spectroscopy is a recent technique that combines the nanometric resolution of the AFM probe with the identification of functional groups from IR spectroscopy. ${ }^{15}$ The AFM-IR lateral resolution is determined by the radius of the AFM tip, which is generally less than $20 \mathrm{~nm} . .^{15,16}$ The axial resolution of this technique strongly depends on the thermal characteristics of the sample. Low thermal conductivity and thermal expansion induced by light absorption in the sample can limit the depth resolution. The penetration depth of the infrared laser also depends on the physical characteristics of the sample. ${ }^{17}$ Typically, a minimum sample thickness is necessary (generally around $15 \mathrm{~nm}$ ) to produce detectable cantilever deflections. ${ }^{16,17}$ Therefore, the AFM-IR spectroscopy can detect smaller domains than Raman microspectroscopy by at least one order of magnitude. This technique has been shown to be powerful to reveal the presence of domains in polymeric and composite materials, as well as in drug formulations and cells (for a review, see ref. 15). Recently, Marcott et al. ${ }^{18}$ have used this technique to characterize the distribution of corneocyte proteins and intercellular lipids on a cross-section of human SC. They were able to identify regions with high lipid content at the perimeter of the corneocytes, using the characteristic absorption of the $\nu \mathrm{CH}$ region.

In the present work, we are exploiting the spatial resolution and the chemical imaging capabilities of AFM-IR spectroscopy to gain insights into lipid spatial distribution in SC model mixtures; this approach provides chemical imaging of SC model mixtures at, to our knowledge, an unprecedented spatial resolution. More specifically, we took advantage of the spatial resolution of the AFM-IR technique to attempt characterizing the fluid phase associated with Cer EOS. We characterized an SC model mixture of Cer NS, Cer EOS, four FFAs with acyl chain length varying from 16 to 24 carbon atoms, and Chol. The molecular structure of each component is presented in Fig. 1.

The three lipid species (Cer, FFAs, and Chol) were in an equimolar ratio. The molar ratio between Cer NS and Cer EOS was 0.6/0.4. The fatty acid mixture included lignoceric acid (FFA24), arachidic acid (FFA20), stearic acid (FFA18), and palmitic acid (FFA16) in a molar ratio of 84.6/8.75/4.57/2.05; this distribution is representative of the SC FFA composition. ${ }^{19}$ We took advantage of the isotopic shift and prepared mirror mixtures: mixtures with the same composition but that included a different deuterated component. This strategy allowed us to probe the behavior of individual lipids in a relatively complex mixture.

\section{Materials and methods}

\section{Materials}

$\mathrm{N}$-melissoyl-oleoyloxy hexacosanoyl-D-erythro-sphingosine (Cer EOS, $>91 \%$ ) and N-lignoceroyl-D-erythro-sphingosine (Cer NS, >96\%) were generously provided by Evonik (Essen Germany). Partly deuterated Cer EOS (Cer EOS- $d$ ) (>99\%) and Cer NS (Cer NS- $d$ ) (>99\%) were custom 
synthesized by Evonik (Essen, Germany). FFA16, FFA18, FFA20, FFA24, and Chol, all with a purity $>98 \%$, were obtained from SigmaAldrich Chemie GmbH (Schnelldorf, Germany). Deuterated FFA24 (FFA24- $d$ ) (>98\%) was obtained from ARC Laboratories (Apeldoorn, Netherlands). Silicon wafers were obtained from Okmetic (Vantaa, Finland). The analytical-grade solvents were supplied by Labscan (Dublin, Ireland).

\section{Mixture preparation}

In this study, we prepared three mirror mixtures: Cer NS/Cer EOS/FFAs-d/Chol, containing deuterated FFA24, Cer NS-d/Cer EOS/ FFAs/Chol, containing Cer NS- $d$, and Cer NS/Cer EOS- $d /$ FFAs/Chol, containing Cer EOS- $d$. To prepare the mixtures, the appropriate amount of lipids ( $\sim 0.6 \mathrm{mg} / \mathrm{sample})$ was dissolved in a chloroform/ methanol $(2: 1 v / v)$ mixture at a concentration of $5 \mathrm{mg} / \mathrm{mL}$. Subsequently, the lipids were sprayed on a silicon substrate using a Camag Linomat IV sample applicator (Muttenz, Switzerland). Spraying was performed at a rate of $5 \mu \mathrm{L} / \mathrm{min}$, under a gentle stream of nitrogen gas. Then, the lipid mixtures were equilibrated for $10 \mathrm{~min}$ at $70{ }^{\circ} \mathrm{C}$, close to the melting temperature of the lipid mixture. After equilibration, the samples were slowly cooled down.

\section{Raman microspectroscopy analysis}

The samples used for the Raman analysis were incubated at $37^{\circ} \mathrm{C}$ for 7 days to ensure a thermodynamically stable state. Then, the samples (waxy solids) were placed on a glass coverslip and gently squeezed to obtain a relatively flat surface. They were subsequently immersed in acetate buffer $(20 \mathrm{mmol} / \mathrm{L}, \mathrm{pH} 5)$ to ensure complete hydration during the measurements. The temperature was adjusted to $33^{\circ} \mathrm{C}$. The Raman spectra were obtained with a Witec Alpha 3000 confocal Raman microscope (Ulm, Germany), equipped with a $63 \times$ immersion objective (NA 1.0), and a diffraction grating of 600 lines $/ \mathrm{mm}$. A $532 \mathrm{~nm}$ laser was used as excitation source with a $10 \mathrm{~mW}$ power at the sample surface. All the images were obtained over a $40 \mu \mathrm{m} \times 40 \mu \mathrm{m}$ area, with a resolution of $0.67 \mu \mathrm{m} /$ pixel and an integration time of $5 \mathrm{~s}$ per spectrum, leading to a total acquisition time of $\sim 2 \mathrm{~h}$. The false-colour distribution maps and histograms were generated using the OriginPro software.

\section{AFM-IR analysis}

The samples used for the AFM-IR analysis were incubated directly on the silicon substrate in a $100 \%$ humidity chamber, at $37{ }^{\circ} \mathrm{C}$ for $16 \mathrm{~h}$. The thickness of the samples was at least $25 \mu \mathrm{m}$, with a surface roughness of $110 \pm 50 \mathrm{~nm}$ (mean roughness, Ra). The AFM-IR measurements were performed on a NanoIR2 system from Bruker (Santa Barbara, USA), using a gold-plated silicon nitride AFM probe with an elastic constant of $0.07-0.4 \mathrm{~N} / \mathrm{m}$ and nominal radius of $20 \mathrm{~nm}$ (model PR-EX-nIR2, Bruker, Santa Barbara, USA). The nanoscale IR spectra were collected from the surface of the mixtures between 2000 and $2250 \mathrm{~cm}^{-1}$ for the $\nu \mathrm{CD}$ region and between 2700 and $3050 \mathrm{~cm}^{-1}$ for the $\nu \mathrm{CH}$ region. The spectral resolution was set to $4 \mathrm{~cm}^{-1}$, and 2048 scans were co-averaged. The AFM-IR images derived from the IR absorption band intensities measured at two different wavelengths $\left(2190 \mathrm{~cm}^{-1}\right.$ for $\nu \mathrm{CD}$ region and $2916 \mathrm{~cm}^{-1}$ for $\nu \mathrm{CH}$ region) over an area of at least $5 \mu \mathrm{m} \times 5 \mu \mathrm{m}$, with a scan rate of $0.1 \mathrm{~Hz}$ and a resolution of $20 \mathrm{~nm} /$ pixel, using 16 co-averages. The laser power limit was kept within $0.1 \%-0.4 \%$, with a resonant frequency of $65 \mathrm{kHz}$. All measurements were performed at room temperature. The distribution maps and histograms were obtained using the OriginPro software.

\section{Results and discussion}

Figure 2 shows the Raman spectra of the three mirror mixtures. These were obtained from averaging those recorded for the Raman imaging and, therefore, are representative of the entire surface.
Fig. 2. Raman spectra of the (A) Cer EOS/Cer NS/FFAs-d/Chol, (B) Cer EOS/Cer NS- $d /$ FFAs/Chol, and (C) Cer EOS-d/Cer NS/FFAs/Chol mixtures.

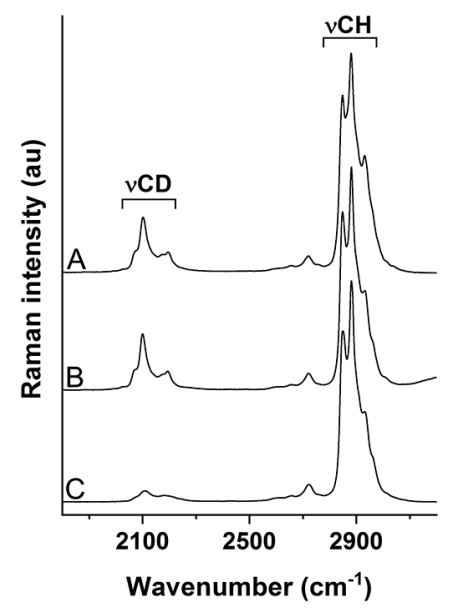

The $\nu \mathrm{CD}$ bands assigned to the deuterated lipid vibrational modes were located between 2000 and $2250 \mathrm{~cm}^{-1}$. This signal was mainly composed by the symmetric $\left(\nu_{\mathrm{s}} \mathrm{CD}_{2}\right)$ and antisymmetric $\left(\nu_{\mathrm{as}} \mathrm{CD}_{2}\right)$ methylene stretching modes at 2100 and $2175 \mathrm{~cm}^{-1}$, respectively, and by the symmetric $\left(\nu_{\mathrm{s}} \mathrm{CD}_{3}\right)$ and antisymmetric $\left(\nu_{\mathrm{as}} \mathrm{CD}_{3}\right)$ methyl stretching modes, with components at 2120 and $2075 \mathrm{~cm}^{-1}$, respectively. ${ }^{12,20}$ The width of the $\nu_{\mathrm{s}} \mathrm{CD}_{2}$ band can be used to characterize the deuterated chain order. ${ }^{12}$ The bandwidth measured at $65 \%$ of the height was $24.4 \mathrm{~cm}^{-1}$ for the mixture containing FFA24- $d, 22.4 \mathrm{~cm}^{-1}$ for that with deuterated Cer NS- $d$, and $43.5 \mathrm{~cm}^{-1}$ for that with deuterated oleate chain in Cer EOS. These results agree well with those previously reported ${ }^{9}$ and indicate that the FFA24 and Cer NS acyl chains were ordered, whereas the oleate chain in Cer EOS was highly disordered.

The $\nu \mathrm{CH}$ bands were observed between 2780 and $3100 \mathrm{~cm}^{-1}$. The symmetric $\left(\nu_{\mathrm{s}} \mathrm{CH}_{2}\right)$ and antisymmetric $\left(\nu_{\mathrm{as}} \mathrm{CH}_{2}\right)$ stretching modes were located at 2848 and $2881 \mathrm{~cm}^{-1}$, respectively. The component at $2933 \mathrm{~cm}^{-1}$ is assigned to a $\mathrm{CH}_{3}$ stretching mode. ${ }^{21,22}$ The intensity ratio of the $\nu_{\mathrm{s}} \mathrm{CH}_{2}$ and $\nu_{\mathrm{as}} \mathrm{CH}_{2}$ bands $\left(\mathrm{I}_{2881} / \mathrm{I}_{2848}\right)$ can be used to characterize the chain order of the hydrogenated lipids. The Raman spectra of the three mixtures showed an $I_{2881} / I_{2848}$ ratio of 1.3; this value is typical of ordered hydrogenated chains of lipids. ${ }^{22,23}$ It is proposed that the contribution of the highly disordered hydrogenated Cer EOS is too small (less than $30 \%$ of the methylene groups of Cer EOS were deuterated, and this lipid only represented 13 (mol)\% of the total lipid amount) to significantly affect the $I_{2881} / I_{2848}$ ratio.

To investigate the FFA spatial distribution in the mixtures, we recorded 3600 Raman spectra over a $40 \mu \mathrm{m} \times 40 \mu \mathrm{m}$ surface. For the set-up that was used $(\lambda=523 \mathrm{~nm}, \mathrm{NA}=1)$, the focus was made at the lipid-buffer interface, and the depth of focus was estimated to $2 \mu \mathrm{m}$. The spectra were treated to obtain false-colour distribution maps of the lipid molar proportion. First, the $\nu$ CD (2000$\left.2250 \mathrm{~cm}^{-1}\right)$ and $\nu \mathrm{CH}\left(2780-3100 \mathrm{~cm}^{-1}\right)$ regions were integrated for each spectrum. The areas of the $\nu \mathrm{CD}$ and $\nu \mathrm{CH}$ regions were proportional, respectively, to the $\mathrm{CD}$ and $\mathrm{CH}$ local concentration. However, because the Raman activity is not the same for the two regions, we pose the following equation:

$$
\frac{A_{\mathrm{CD}}}{A_{\mathrm{CH}}}=f \frac{[\mathrm{CD}]}{[\mathrm{CH}]}
$$

where $A_{\mathrm{CD}}$ and $A_{\mathrm{CH}}$ are the mean values of the $\nu \mathrm{CD}$ and $\nu \mathrm{CH}$ areas, respectively, $[\mathrm{CD}]$ and $[\mathrm{CH}]$ are the molar proportions of the $\mathrm{CD}$ and $\mathrm{CH}$ lipids in the mixture, respectively, and $f$ is a constant equal to the ratio of the response factors for the $\mathrm{CD}$ and $\mathrm{CH}$ region. 
Fig. 3. Raman images and corresponding histograms of the (A) Cer EOS/Cer NS/FFAs- $d / C h o l$, (B) Cer EOS/Cer NS-d/FFAs/Chol, and (C) Cer EOS- $d / C e r$ NS/FFAs/Chol mixtures. The histograms combined the data of two independent maps from each mixture and therefore represent 7200 pixels. [Colour online.]
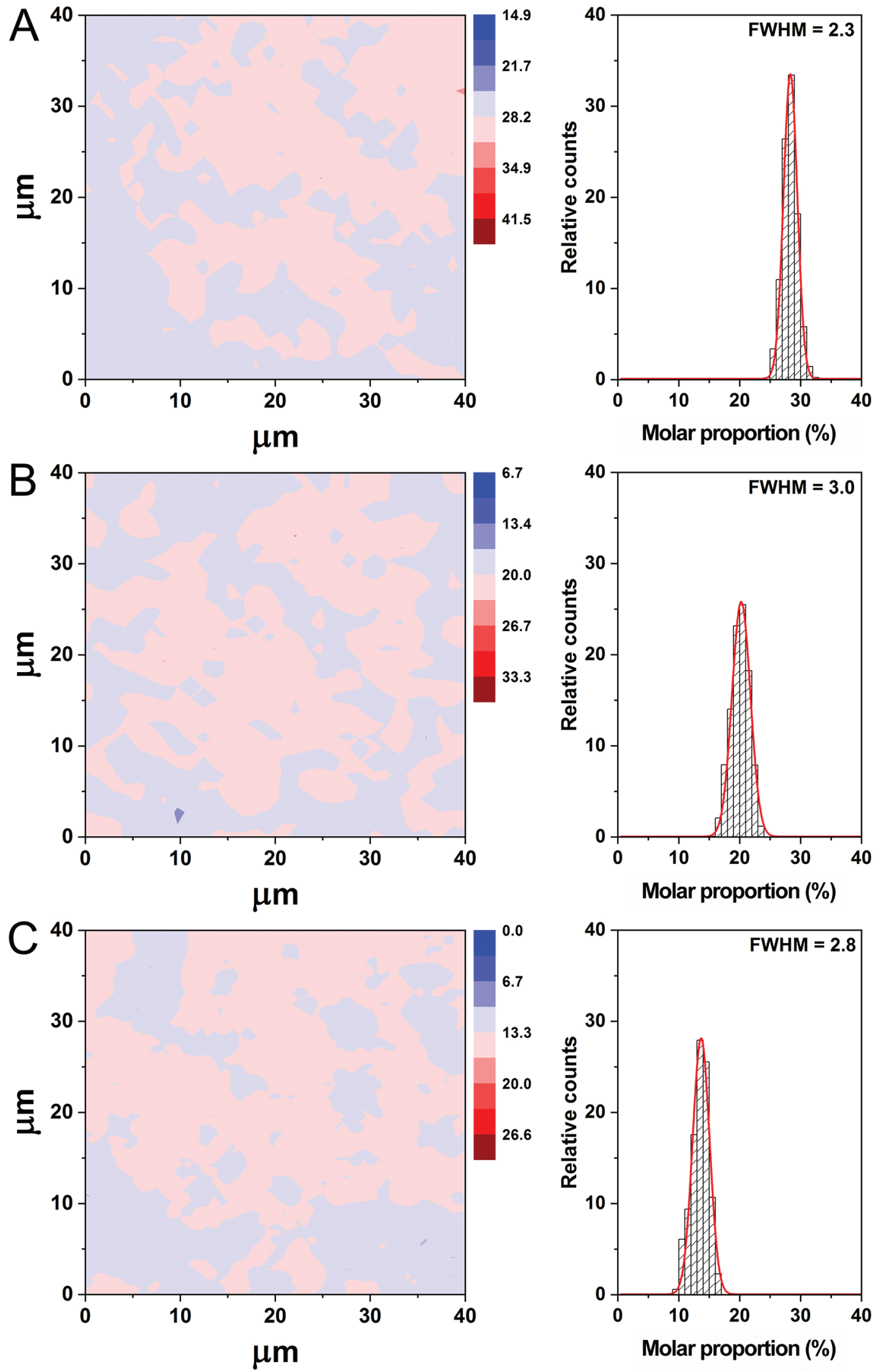

- Published by NRC Research Press 
We estimated $f$ from the spectrum averaged over the whole investigated area:

$$
f=\frac{A_{\mathrm{CD}}^{\mathrm{av}}}{A_{\mathrm{CH}}^{\mathrm{av}}} \frac{[\mathrm{CH}]_{\mathrm{mix}}}{[\mathrm{CD}]_{\text {mix }}}
$$

where $A_{\mathrm{CD}}^{\mathrm{av}}$ and $A_{\mathrm{CH}}^{\mathrm{av}}$ are, respectively, the areas of the $\nu \mathrm{CD}$ region and of the $\nu \mathrm{CH}$ region of the average spectrum, and $[\mathrm{CD}]_{\text {mix }}$ and $[\mathrm{CH}]_{\text {mix }}$ are, respectively, the proportions of deuterated species and of hydrogenated species in the mixture.

The molar ratio associated with a given pixel is given as follows:

$$
\frac{[\mathrm{CH}]^{\mathrm{pix}}}{[\mathrm{CD}]^{\mathrm{pix}}}=f \frac{A_{\mathrm{CH}}^{\mathrm{pix}}}{A_{\mathrm{CD}}^{\mathrm{pix}}}
$$

The proportion of the deuterated species $\mathrm{X}_{\mathrm{CD}}^{\mathrm{pix}}$ is given as follows:

$$
X_{\mathrm{CD}}^{\mathrm{pix}}=\left(\frac{[\mathrm{CD}]^{\mathrm{pix}}}{[\mathrm{CD}]^{\mathrm{pix}}+[\mathrm{CH}]^{\mathrm{pix}}}\right) \times 100
$$

Combining eqs. 2-4, it is possible to obtain the molar proportion of each pixel:

$$
X_{\mathrm{CD}}^{\mathrm{pix}}=\left[1 \mid\left(1+\frac{A_{\mathrm{CH}}^{\mathrm{pix}}}{A_{\mathrm{CD}}^{\mathrm{pix}}} \frac{[\mathrm{CH}]_{\mathrm{mix}} A_{\mathrm{CD}}^{\mathrm{av}}}{[\mathrm{CD}]_{\mathrm{mix}} A_{\mathrm{CH}}^{\mathrm{av}}}\right)\right] \times 100
$$

The resulting chemical images and their corresponding histograms are presented in Fig. 3.

The spatial distribution of the three deuterated lipids over the investigated areas by Raman spectroscopy was very similar for all samples. The distributions for FFA24, Cer NS, and Cer EOS were centered at 28.2, 20.0, and 13.3, respectively; these values corresponded well to their molar proportions in the mixture. The molar proportions calculated for the pixels over the investigated region could be well represented by normal distributions. The full width at half maximum (FWHM) of these normal distributions of the three mirror mixtures were between 2.3 and 3.0, indicating that the distribution of three components is rather similar and homogeneously over the surface.

Infrared spectroscopy provides specific information relative to the conformational order of the acyl chains of deuterated lipid in complex mixtures by analyzing the $\nu \mathrm{CD}$ region. Figure 4 compares the $\nu$ CD region of the AFM-IR spectra of the three mixtures under study. This region is dominated by the $\nu_{\mathrm{s}} \mathrm{CD}_{2}$ mode at $\sim 2090 \mathrm{~cm}^{-1}$ and the $\nu_{\text {as }} \mathrm{CD}_{2}$ mode at $\sim 2200 \mathrm{~cm}^{-1}$. It is well established that the position of the $\nu_{\mathrm{s}} \mathrm{CD}_{2}$ mode is sensitive to the conformational order of hydrocarbon chains. ${ }^{24,25}$ The AFM-IR technique makes this approach applicable at the nanometer scale. It is generally accepted that lipids in the solid phase, where all chains are in all-trans conformation, display a $\nu_{\mathrm{s}} \mathrm{CD}_{2}$ band under $2090 \mathrm{~cm}^{-1}$, whereas acyl chains in the liquid phase, where a considerable number of gauche conformations exist, typically exhibit a $\nu_{\mathrm{s}} \mathrm{CD}_{2}$ band at around $2094 \mathrm{~cm}^{-1} .{ }^{26}$

In the Cer EOS/Cer NS/FFAs-d/Chol and Cer EOS/Cer NS-d/FFAs/ Chol mixtures, the position of the $v_{\mathrm{s}} \mathrm{CD}_{2}$ band was located at $2084 \mathrm{~cm}^{-1}$, confirming the highly ordered FFA24- $d$ and Cer NS- $d$ acyl chains. ${ }^{27}$ In contrast, the position of the $\nu_{\mathrm{s}} \mathrm{CD}_{2}$ in the Cer EOS-d/Cer NS/FFAs/Chol sample was observed at $2093 \mathrm{~cm}^{-1}$, indicating that the oleate chain of Cer EOS was highly disordered, a result that is in very good agreement with previous findings., ${ }^{9,28}$ AFM-IR spectra were recorded from different locations of the samples and no significant difference was observed.
Fig. 4. Typical $\nu$ CD region of the AFM-IR spectra from the (top) Cer EOS-d/Cer NS/FFAs/Chol, (middle) Cer EOS/Cer NS-d/FFAs/Chol, and (bottom) Cer EOS/Cer NS/FFAs-d/Chol mixtures. [Colour online.]

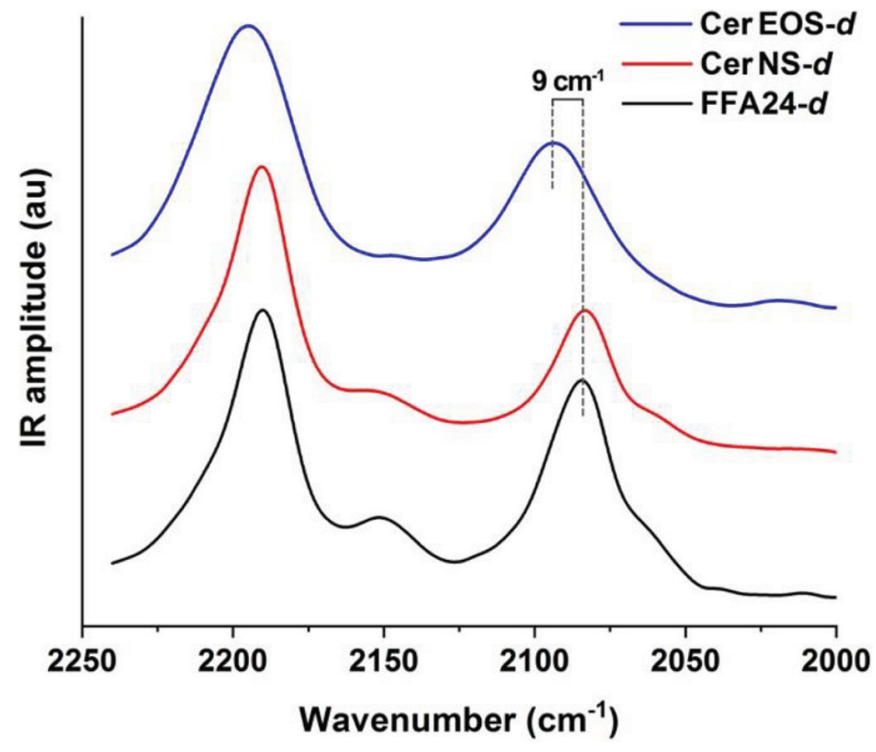

The AFM-IR spectroscopy was used to determine the lipid spatial distribution in the model membranes at the nanometer scale and assess whether the liquid hydrocarbon phase formed by the oleate chain of Cer EOS could be specifically located.9,28 We recorded the signal intensity at $2190 \mathrm{~cm}^{-1}\left(\nu_{\mathrm{as}} \mathrm{CD}_{2}\right)$ and $2916 \mathrm{~cm}^{-1}$ $\left(\nu_{\mathrm{as}} \mathrm{CH}_{2}\right)$ over a $5 \mu \mathrm{m} \times 5 \mu \mathrm{m}$ surface, with steps of $20 \mathrm{~nm} /$ pixel. The measured infrared intensities were treated using a similar procedure as for Raman images to obtain the AFM-IR false-colour images (Fig. 5); the band areas were replaced by the infrared intensities at the two specific wavelengths.

The mean values of the molar proportion over the surface for FFA24- $d$, Cer NS- $d$, and Cer EOS- $d$ were $28.3 \%, 19.9 \%$, and $13.5 \%$, respectively. These values agreed well with the molar proportions of these lipids in the mixture. The maps obtained at this enhanced spatial resolution revealed that the three mixtures were found mostly homogeneous, with the molar proportions varying only by $\pm 3.3 \%$. However, small slightly enriched and depleted regions with submicron dimensions could be observed. The Cer EOS/Cer NS/ FFAs-d/Chol mixture (Fig. 5A) showed FFA24-enriched areas where the molar proportion increased up to $35 \%$ and FFA24-depleted zones where it was $18 \%$. The Cer EOS/Cer NS- $d /$ FFAs/Chol sample (Fig. 5B) also showed Cer NS-enriched regions where the molar proportion reached $27 \%$, in contrast with the Cer NSdepleted zones, where the values decreased to $13 \%$. Similarly, the Cer EOS- $d /$ Cer NS/FFAs/Chol mixture (Fig. 5C) also showed the coexistence of Cer EOS-enriched and Cer EOS-depleted zones, with molar proportions of $27 \%$ and $7 \%$, respectively. The histograms, combining the values of at least three different AFM-IR maps, illustrate the individual lipid distribution over the surface.

The proportion distributions could be reproduced by normal distributions. Their FWHM varied between $2.5 \%$ and $3.7 \%$, indicating a rather homogeneous spatial distribution of the deuterated components, even at this nanoscopic scale. We performed a statistical analysis of the data, looking for outliers. The outliers were defined as values that lie at an abnormal distance from the average values, and in our analysis, they would correspond to lipidenriched and lipid-depleted regions. The outlier plots obtained for each mixture (Supplementary Fig. S1) showed that the Raman microspectroscopy results include almost all the experimental data with three standard deviations from the mean (that should include $99.7 \%$ of the points). In contrast, the analysis from the AFM-IR data showed the presence of a few outliers, suggesting 
Fig. 5. AFM-IR images and corresponding histograms of the (A) Cer EOS/Cer NS/FFAs- $d / C h o l$, (B) Cer EOS/Cer NS- $d /$ FFAs/Chol, and (C) Cer EOS- $d /$ Cer NS/FFAs/Chol mixtures. The images in the left column correspond to those that showed the most pronounced heterogeneity, whereas those in the middle column were the most homogeneous (out of at least three maps). The histograms combined the data of all the examined maps and are derived from at least 260000 pixels. [Colour online.]
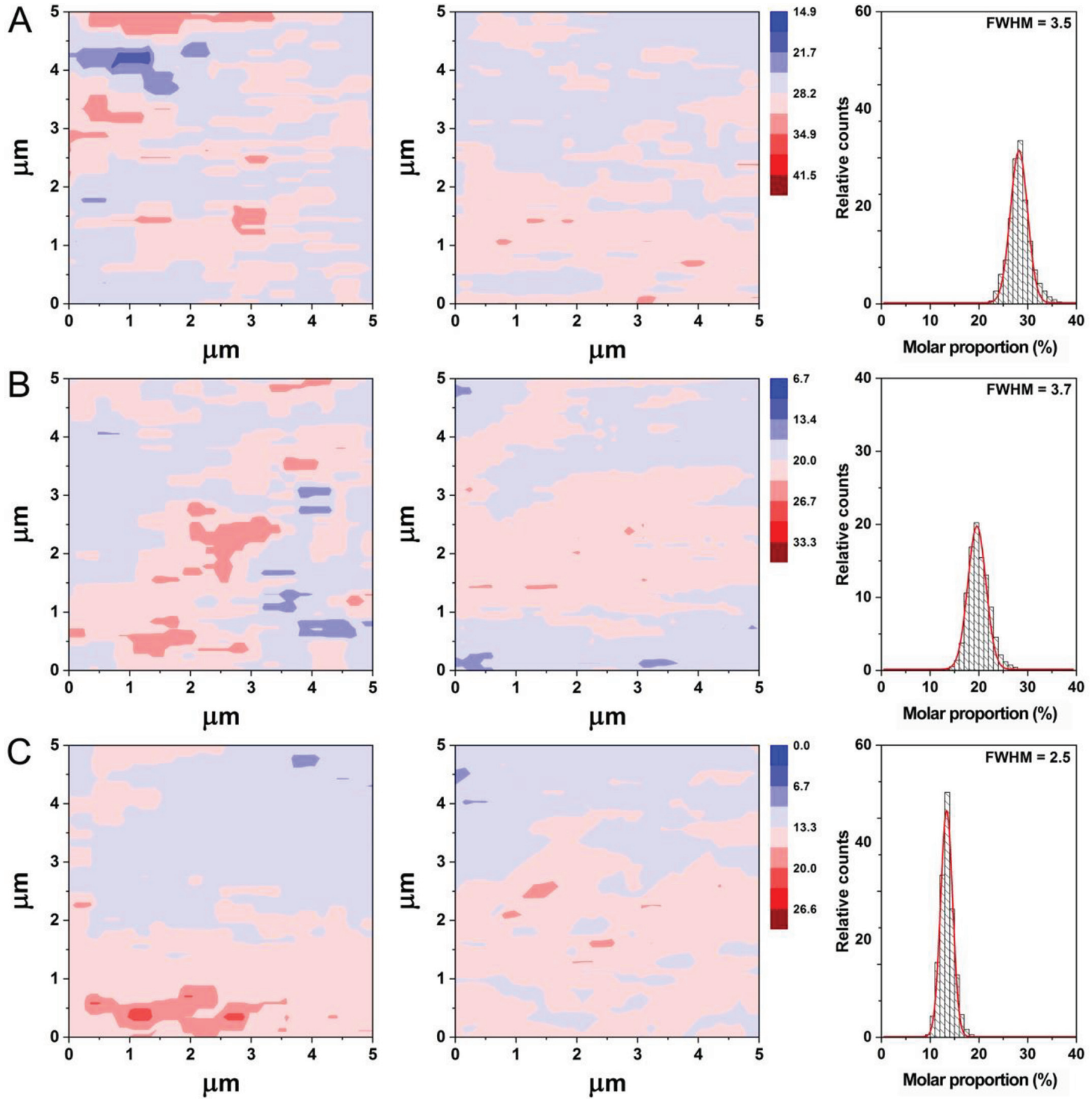

that some chemical heterogeneity can be identified at the nanoscopic length scale. Moreover, these data points formed small clusters (see on the maps, Fig. 5), supporting the idea that they correspond to small regions of unusual chemical composition rather than single spikes. These results show that the gain of spatial resolution by a factor of about 50 associated with the AFM-IR technique relative to Raman microspectroscopy provides a more detailed chemical mapping. In the specific case of the investigated SC model mixture, lipid-enriched and lipid-depleted zones could be observed only when increasing the spatial resolu- tion. At this point, it is not established whether the formation of these regions is the result of a thermodynamic phase separation or a consequence of the sample preparation. The presence of phase-separated cholesterol has been previously observed in this model mixture. ${ }^{9,10}$ These small cholesterol crystals could partly contribute to the observation of $\mathrm{CH}$-enriched regions, as such crystals formed of pure cholesterol would boost the $\nu \mathrm{CH}$ IR signal measured at that location.

Overall, the AFM-IR results reveal that most of the lipid phase is rather homogenous from a chemical composition point of view, 
as well as from the point of view of a chain order composition: the oleate chain of Cer EOS appears to be systematically highly disorder, whereas the other lipid components are highly ordered. Therefore, this finding excludes extensive phase separations at the $20 \mathrm{~nm}$ scale. These findings are consistent with the model ${ }^{9-11}$ proposing that these oleate nanodrops are concentrated in the central layer (of $\sim 4 \mathrm{~nm}$ thick) of the three-layer organization of the LPP, which is still far from the resolution of the AFM-IR spectroscopy technique.

In conclusion, this work shows that AFM-IR spectroscopy can provide valuable information about the lipid mixing properties in SC model membranes at the nanoscale. The lack of observable oleate nanodrops in this SC model membrane is consistent with the proposed three-layer model, which predicts liquid domains formed by Cer EOS unsaturated chain at a size smaller than the current detection limit of the technique.

\section{Supplementary data}

Supplementary data are available with the article through the journal Web site at http://nrcresearchpress.com/doi/suppl/10.1139/ cjc-2019-0471.

\section{Acknowledgements}

This work was supported by the Natural Sciences and Engineering Research Council of Canada and by the Fonds de Recherche du Québec - Nature et Technologies (FQRNT) through its financial support to the Centre québécois sur les matériaux fonctionnels (CQMF). Evonik Industries AG (Essen, Germany) is gratefully acknowledged for the generous donation of the hydrogenated and deuterated ceramides.

\section{References}

(1) Elias, P. M. J. Invest. Dermatol. 1983, 80 (1, Suppl.), S44. doi:10.1038/jid.1983.12.

(2) Bouwstra, J. A.; Gooris, G. S.; van der Spek, J. A.; Bras, W. J. Invest. Dermatol. 1991, 97 (6), 1005. doi:10.1111/1523-1747.ep12492217.

(3) Mojumdar, E. H.; Gooris, G. S.; Barlow, D. J.; Lawrence, M. J.; Deme, B.; Bouwstra, J. A. Biophys. J. 2015, 108 (11), 2670. doi:10.1016/j.bpj.2015.04.030.

(4) Rabionet, M.; Gorgas, K.; Sandhoff, R. Biochim. Biophys. Acta 2014, 1841 (3), 422. doi:10.1016/j.bbalip.2013.08.011.
(5) Weerheim, A.; Ponec, M. Arch. Dermatol. Res. 2001, 293 (4), 191. doi:10.1007| s004030100212.

(6) t'Kindt, R.; Jorge, L.; Dumont, E.; Couturon, P.; David, F.; Sandra, P.; Sandra, K. Anal. Chem. 2012, 84 (1), 403. doi:10.1021/ac202646v.

(7) de Jager, M. W.; Gooris, G. S.; Dolbnya, I. P.; Bras, W.; Ponec, M.; Bouwstra, J. A. J. Lipid Res. 2004, 45 (5), 923. doi:10.1194/jlr.M300484-JLR200.

(8) Bouwstra, J. A.; Gooris, G. S.; Dubbelaar, F. E. R.; Ponec, M. J. Invest. Dermatol. 2002, 118 (4), 606. doi:10.1046/j.1523-1747.2002.01706.x.

(9) Paz Ramos, A.; Gooris, G.; Bouwstra, J.; Lafleur, M. J. Lipid Res. 2018, 59 (1), 137. doi:10.1194/jlr.M080432.

(10) Mojumdar, E. H.; Gooris, G. S.; Groen, D.; Barlow, D. J.; Lawrence, M. J: Demé, B.; Bouwstra, J. A. Biochim. Biophys. Acta 2016, 1858 (8), 1926. doi:10. 1016/j.bbamem.2016.05.006.

(11) Wang, E.; Klauda, J. B. J. Am. Chem. Soc. 2019, 141 (42), 16930. doi:10.1021/jacs. $9 \mathrm{~b} 08995$.

(12) Mendelsohn, R.; Sunder, S.; Bernstein, H. J. Biochim. Biophys. Acta 1976, 443 (3), 613. doi:10.1016/0005-2736(76)90480-6.

(13) Dieing, T.; Hollricher, O.; Toporski, J. Confocal raman microscopy. Springer: Berlin (2011).

(14) Everall, N. J. Appl. Spectrosc. 2000, 54 (10), 1515. doi:10.1366/0003702001948439.

(15) Dazzi, A.; Prater, C. B. Chem. Rev. 2017, 117 (7), 5146. doi:10.1021/acs.chemrev. $6 \mathrm{~b} 00448$.

(16) Handschuh-Wang, S.; Wang, T.; Zhou, X. RSC Adv. 2017, 7 (75), 47464. doi: 10.1039/C7RA08515J.

(17) Lahiri, B.; Holland, G.; Centrone, A. Small 2013, 9 (3), 439. doi:10.1002/smll. 201200788.

(18) Marcott, C.; Lo, M.; Kjoller, K.; Domanov, Y.; Balooch, G.; Luengo, G. S. Exp. Dermatol. 2013, 22 (6), 419. doi:10.1111/exd.12144.

(19) Wertz, P. Epidermal lipids. In Physiology, Biochemistry and Molecular Biology of the Skin. Goldsmith, L. A., Editor. Oxford University Press: Oxford (1991): p. 205.

(20) Bryant, G. J.; Lavialle, F.; Levin, I. W. J. Raman Spectrosc. 1982, 12 (2), 118. doi:10.1002/jrs.1250120205.

(21) Gaber, B. P.; Peticolas, W. L. Biochim. Biophys. Acta 1977, 465 (2), 260. doi:10. 1016/0005-2736(77)90078-5.

(22) Snyder, R. G.; Scherer, J. R.; Gaber, B. P. Biochim. Biophys. Acta 1980, 601, 47. doi:10.1016/0005-2736(80)90512-X.

(23) Lafleur, M.; Samson, I.; Pézolet, M. Chem. Phys. Lipids 1991, 59 (3), 233. doi:10. 1016/0009-3084(91)90023-5.

(24) Mendelsohn, R.; Moore, D. J. Chem. Phys. Lipids 1998, 96 (1-2), 141. doi:10.1016/ S0009-3084(98)00085-1.

(25) Moore, D. J.; Rerek, M. E.; Mendelsohn, R. J. Phys. Chem. B 1997, 101 (44), 8933. doi:10.1021/jp9718109.

(26) Gooris, G. S.; Bouwstra, J. A. Biophys. J. 2007, 92 (8), 2785. doi:10.1529/biophysj. 106.094292.

(27) Mendelsohn, R.; Flach, C. R.; Moore, D. J. Biochim. Biophys. Acta 2006, 1758 (7), 923. doi:10.1016/j.bbamem.2006.04.009.

(28) Janssens, M.; Gooris, G. S.; Bouwstra, J. A. Biochim. Biophys. Acta 2009, 1788 (3), 732. doi:10.1016/j.bbamem.2009.01.003. 